Pacific Journal of Mathematics

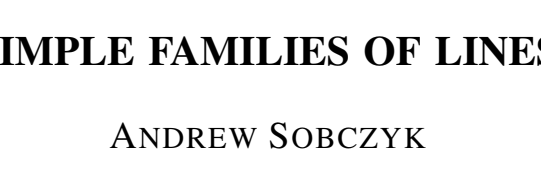




\section{SIMPLE FAMILIES OF LINES}

\section{ANDREW SOBCZYK}

1. Introduction. Planar families of lines are studied by P. C. Hammer and the author in [2], and families of lines in the plane and in ordinary space by the author in [6]. Families of lines in vector spaces $E_{3}$ and $E_{n}$ are mentioned in connection with convex bodies in [1]. The present paper gives a classification of simple types of families in $(n+1)$ dimensional real vector space $E_{n+1}$. Theorems are obtained on relations between the type of the family $F$, and the properties which $F$ may possess, of containing exactly one line in every direction, and of simply or multiply covering the points of $E_{n+1}$.

2. Notation and definitions. With respect to an $n$ dimensional vector subspace $E_{n}$ of $(n+1)$ dimensional real vector space $E_{n+1}$ a line $L$ in $E_{n+1}$ will be called horizontal if it is parallel to $E_{n}$. Any family $F$ of non-horizontal lines in $E_{n+1}$, for which there is a hyperplane $H$ parallel to $E_{n}$ such that each point of $H$ is covered exactly once by $F$, determines a single valued function $y=f(x)$ on $H$ to any parallel hyperplane $K: x, y$ are the points in which the line $L$ of $F$ which covers $x$ intersects $H, K$. Corresponding to any basis in $E_{n}$, and choice of origins in $H, K$, the function $f(x)$ will be represented by real valued functions $y_{i}=f_{i}\left(x_{1}, \cdots, x_{n}\right), i=1, \cdots, n$. (For definiteness, let $E_{n+1}$ be Euclidean, and choose the origins in $H, K$ to be their points of intersection with the line through the common origin of $E_{n+1}, E_{n}$, which is orthogonal to $E_{n}$.)

A family $F$ will be said to be composed of two lower dimensional associated families, $F_{p}$ and $F_{n-p}$, if there is a choice of basis such that the $n$ real functions have the form $y_{i}=f_{i}\left(x_{1}, \cdots, x_{p}\right), i=1, \cdots, p ; y_{j}=$ $f_{j}\left(x_{p+1}, \cdots, x_{n}\right), j=p+1, \cdots, n$. (The dimension of an associated family of course is one greater than the subscript; thus for example a three dimensional family may be composed of two associated two dimensional families.)

A family $F$ is primary if it contains exactly one line in every nonhorizontal direction, representative if it contains exactly one line in every direction. We say that a family $F$ of lines is simple if every point of $E_{n+1}$ is covered exactly once by the family; outwardly simple if every point exterior to some sphere $S_{n}$ has the same property in relation to the family. If the distances from the origin of the lines of an outwardly simple family are bounded, then for a sufficiently large sphere $S_{n}$, if $P, g(P)$ are the points in which the line $L$ of $F$ covering $P$ pierces $S_{n}$,

Received June 30, 1954. 
the transformation $g$ is an involutory mapping of $S_{n}$ into itself which has no fixed point. By the theorem proved in [4], if $g$ is continuous, such an outwardly simple family covers the interior of $S_{n}$ and therefore covers all of $E_{n+1}$. Note the difference in the present usage of the term outwardly simple, and the usage in [2], [1] (where in order that $F$ be called outwardly simple the additional requirements are made that $F$ is representative, and that the corresponding involutory transformation $g$ of $S_{n}$ into itself is continuous and has no fixed point.)

3. Stacks and sheafs. In case the lines of $F$ are all contained in the $p$-sheaf of all $p$-flats in $E_{n+1}$ parallel to a fixed $p$ dimensional vector subspace $E_{p}, F$ will be called a $p$-stack, $1 \leqq p \leqq n$. If $p=1$, the 1 -stack or 1-sheaf $F$ is a simple sheaf of parallel lines in $E_{n+1}$. A $p$-stack $F$ may be such that lines of the sub-family, for each of the parallel $p$ flats $R_{p}$, are contained in $(p-1)$-flats of a $(p-1)$-sheaf in $R_{p}$; such a family may be called a $p,(p-1)$-stack. A family $F$ is a $p,(p-1), \cdots$, $q$-stack if it divides successively into sub-families contained in parallel $p-,(p-1)-, \cdots, q-$, sheafs, where not all of the sub-families in the flats of lowest dimension $q$ are stacks. Evidently a $q$-stack is a $p, \cdots, q$ stack for all $p$ in $q<p \leqq n$. A $k$-stack, for any $k \leqq n$, cannot be a primary family, since the directions of its lines are confined to the directions contained in a $k$ dimensional subspace $\mathrm{E}_{k}$.

A family $F$ of non-horizontal lines is an $n, \cdots,(n-p)$-stack if, with respect to some basis in $E_{n}$, the last $(p+1)$ equations for the family are of the form

$$
\begin{aligned}
y_{n} & =x_{n}+u_{n}, y_{n-1}=x_{n-1}+u_{n-1}\left(x_{n}\right), \cdots, \\
y_{n-p} & =x_{n-p}+u_{n-p}\left(x_{n}, \cdots, x_{n-p+1}\right) .
\end{aligned}
$$

This follows since $y_{k}=x_{k}+c_{k}, c_{k}$ constant, is the equation of a $k$-sheaf in a $(k+1)$-flat, $k=1, \cdots, n$.

4. Linear transformation corresponding to a pencil. Choose a basis in $E_{n+1}$ so that the equations of $H, K$ are respectively $x_{n+1}=a, y_{n+1}=b$. Then points in $H$ may be denoted by $(x ; a)$, in $K$ by $(y ; b)$, and any point in $E_{n+1}$ by $\left(z ; z_{n+1}\right)$, where $x, y, z$ are in $E_{n}$.

We determine the transformation $y=f(x)$ which corresponds to the pencil of lines through a point $\left(w ; w_{n+1}\right), w$ in $E_{n}$, of $E_{n+1}$. Any nonhorizontal line of the pencil has equations

$$
\frac{z_{1}-w_{1}}{m_{1}}=\cdots=\frac{z_{n}-w_{n}}{m_{n}}=\frac{z_{n+1}-w_{n+1}}{m_{n+1}},
$$

where $\left(m_{1}, \cdots, m_{n+1}\right)$ is a non-horizontal $\left(m_{n+1} \neq 0\right)$ unit vector of $E_{n+1}$. 
(Let it be understood that if $m_{k}=0,1 \leqq k \leqq n$, the presence of the ratio $\left(z_{k}-w_{k}\right) / 0$, in this form of the equations for the line, means that $z_{k}=w_{k}$ is one of the equations.) The coordinates of the points of intersection $x, y$ of this line with $H, K$ therefore satisfy the equations

$$
\begin{gathered}
\frac{y_{1}-w_{1}}{m_{1}}=\cdots=\frac{y_{n}-w_{n}}{m_{n}}=\frac{b-w_{n+1}}{m_{n+1}}, \\
\frac{x_{1}-w_{1}}{m_{1}}=\cdots=\frac{x_{n}-w_{n}}{m_{n}}=\frac{a-w_{n+1}}{m_{n+1}},
\end{gathered}
$$

or

$$
\begin{gathered}
\frac{y_{1}-w_{1}}{x_{1}-w_{1}}=\cdots=\frac{y_{n}-w_{n}}{x_{n}-w_{n}}=\frac{b-w_{n+1}}{a-w_{n+1}}, y_{j}-w_{j}=\frac{b-w_{n+1}}{a-w_{n+1}}\left(x_{j}-w_{j}\right), \\
j=1, \cdots, n .
\end{gathered}
$$

Thus the transformation corresponding to the pencil, in vector or matrix form, is

$$
(y-w)=c I(x-w), c=\frac{b-w_{n+1}}{a-w_{n+1}},
$$

where $I$ is the identity matrix. Solving for $w_{n+1}$ in terms of $c$, we obtain

$$
w_{n+1}=\frac{c a-b}{c-1}=a-\frac{b-a}{c-1}
$$

5. Affine families. Equation (4.1) for a pencil suggests consideration of the families corresponding to any linear transformation $(y-w)$ $=T(x-w)$, or to any affine transformation $y=T x+u$, where $T$ may be regarded as the matrix of the transformation, $w, u, y, x$ as column matrices of the coordinates of the corresponding points or vectors in $E_{n}$. Let the family corresponding to $y=T x+u$ be called an affine family. It is shown below that, in case $T$ is singular, hyperplane $K$ may be replaced by a parallel hyperplane such that the matrix $T$ for the family $F$, referred to $H$ and the new hyperplane, is non-singular. In our consideration of affine families, let it be assumed, if necessary, that such a new choice for $K$ always is made.

Let $M$ be a hyperplane parallel to $H, K$. Then for any non-horizontal line, if $x, y, z$ are its points of intersection with $H . K, M$, we have

$$
z-y=d(y-x),
$$

or

$$
z=(1+d) y-d x=[(1+d) T-d I] x
$$


for some real $d$ uniquely determined by the position of $M$. Referred to $H, M$ instead of to $H, K$, the family of lines is represented by matrix $[(1+d) T-d I]$ instead of by matrix $T$. The eigenvalues of $[(1+d) T$ $-d I]$ are all of the form $\lambda-d /(1+d)$, where $\lambda$ is an eigenvalue of $T$. Since $T$ has only a finite number of different eigenvalues, $d$ may be chosen so that the eigenvalues of $[(1+d) T-d I]$ are all different from zero. That is, in case $T$ is singular, $d$ may be chosen so that the new matrix $[(1+d) T-d I]$ is non-singular.

In the equation (4.1) for a pencil of lines, the multiplier $c$ is never 1 , since $a \neq b$. If $c=0$, the center of the pencil is in $K ; c=\infty$ corresponds to the center being in $H$. Thus the eigenvalues of matrix $c I$ are all real, equal to $c$, and different from 1 .

If one or several eigenvalues of $T$ are equal to 1 , by suitable choice of basis, $T$ may be put in the form $\left(\begin{array}{cc}U & 0 \\ 0 & V\end{array}\right)$, where the eigenvalues of sub-matrix $U$ are all different from 1 , and $V$ is superdiagonal with all diagonal elements equal to 1. (See [5].) Thus the corresponding family is composed of two associated families, one corresponding to a transformation $U$ which has eigenvalues different from 1 , the other being an $s, \cdots, 1$-stack, where $s$, the multiplicity of the eigenvalue 1 of $T$, is the dimension of $V$. The family $F$, by the last paragraph of $\S 3$, accordingly is an $n, \cdots,(n-s+1)$-stack, and is not representative or primary. Consideration of stacks reduces to consideration of lower dimensional families which are not stacks. For affine families which are not stacks, the eigenvalues of $T$ are different from 1 .

To put the equation for an affine family in the form $(y-w)=T(x-w)$, we must have $u=-T w+w$, or $(T-I) w=-u$. This is possible with $w$ $=0$ if $u=0$, or for any $u$ if $|T-I| \neq 0$. In the latter case, 1 is not an eigenvalue of $T$, and a unique solution for $w$ exists for any $u$. This means that for any affine family, not a stack, the vertical line $x=y=w$ $=-(T-I)^{-1} u$ is a central line of symmetry of the family, as in the case of a pencil.

In case of an affine family, not a stack, the eigenvalues of $T$ are all different from zero and from one. If $T$ further is such that its eigenvalues are all real, and corresponding eigenvectors span $E_{n}$, then if the eigenvectors are chosen as the basis, $T$ has diagonal form, and evidently the corresponding family of lines is composed of associated lower dimensional pencils with centers on $x=y=w$, there being one associated pencil for each distinct eigenvalue $t_{j}$, and the heights of the centers are given by $w_{n+1, j}=\left(a t_{j}-b\right) /\left(t_{j}-1\right)$. The dimension of the space of each associated pencil is one greater than the multiplicity of the corresponding eigenvalue $t_{j}$. Such a family will be called a quasi-pencil, with centers $\left\{\left(w ; w_{n+1, j}\right)\right\}$. 
For example, in $E_{3}$ the family $F$ given by the equations

$$
y_{1}=t_{1} x_{1}, y_{2}=t_{2} x_{2}, t_{1} \neq t_{2}, t_{i} \neq 0, \neq 1, i=1,2,
$$

is a quasi-pencil, and may be described as the set of all lines of intersection of planes of the pencil of planes $y_{1}=t_{1} x_{1}$ with planes of the pencil $y_{2}=t_{2} x_{2}$. The lines $z_{1}=0, z_{3}=a+(b-a) /\left(1-t_{1}\right) ; z_{2}=0, z_{3}=a+(b-a) /\left(1-t_{2}\right)$, are infinitely covered by $F$; all other points in the planes $z_{3}=a+(b-a)$ $/\left(1-t_{1}\right), z_{3}=a+(b-a) /\left(1-t_{2}\right)$, are not covered by $F$. Every other point of $E_{3}$ is covered exactly once by $F$. In order to make the quasi-pencil $F$ cover all of space, it may be extended by addition of the horizontal 1 -sheafs of lines of intersection of the pencils of planes with the horizontal planes $z_{3}=a+(b-a) /\left(1-t_{1}\right), z_{2}=a+(b-a) /\left(1-t_{2}\right)$, but because of the infinite covering of the two skew horizontal lines, even the extended quasi-pencil is not outwardly simple.

In case $T$ has a single real eigenvalue $t_{1}, \neq 0, \neq 1$, let the basis be chosen so that $T$ assumes superdiagonal form. If it is impossible to choose the basis so that all elements above the diagonal vanish, let the corresponding family $F$ be called a skew pencil. It may easily be shown that a skew pencil simply covers all points of $E_{n+1}$ except points in the hyperplane $w_{n+1}=\left(a t_{1}-b\right) /\left(t_{1}-1\right)$, and that in this hyperplane, all points outside the $(n-1)$ dimensional flat $R$ of points $\left(w ; w_{n+1}\right)$ where $w_{n}=0$, cannot be covered. If $t_{12}, t_{23}, \cdots, t_{n-1, n}$ are all different from zero, then the $(n-1)$ dimensional flat $R$ is covered by all lines of $F$ through points $\left(x_{1}, x_{2}, \cdots, x_{n} ; a\right)$ in $H$, where $x_{2}, \cdots, x_{n}$ are uniquely determined by $w_{1}$, $\cdots, w_{n-1}$, but $x_{1}$ is arbitrary; therefore in this case $R$ is infinitely covered by $F$. Otherwise a smaller dimensional flat in the hyperplane $w_{n+1}$ $=\left(a t_{1}-b\right) /\left(t_{1}-1\right)$ is infinitely covered, and the rest of the hyperplane is not covered, by $F$.

For example, in $E_{3}$ the family $F$ given by the equations $y_{1}=t_{1} x_{1}$ $+t_{12} x_{2}, y_{2}=t_{1} x_{2}, t_{1} \neq 0, \neq 1, t_{12} \neq 0$, is a skew pencil. The lines of $F$ for fixed $x_{2}$ are the lines of the pencil $\left(y_{1}-w_{1}\right)=t_{1}\left(x_{1}-w_{1}\right)$, where $w_{1}=t_{12} x_{2}$ l $\left(1-t_{1}\right)$, which are in the plane $y_{2}=t_{1} x_{2}$. The coordinates of the center of the planar pencil, for each $x_{2}$, are $\left(w_{1}, 0 ; w_{3}\right)$, where $w_{3}=\left(t_{1} a-b\right) /\left(t_{1}-1\right)$. Thus $F$ may be described as a union of planar pencils, one in each plane of a pencil of planes through the line $z_{2}=0, z_{3}=w_{3}$, the centers of the planar pencils being located on this line at $z_{1}=w_{1}=t_{12} x_{2} /\left(1-t_{1}\right)$. Accordingly the centers move out unboundedly as $x_{2}$ increases or decreases indefinitely. This skew pencil $F$ simply covers all points of $E_{3}$, except that points of the line of centers in the plane $z_{3}=w_{3}$ are infinitely covered, and all other points of the plane are not covered, by $F$.

As shown in [5], in any case when the eigenvalues of $T$ are all real, by a suitable choice of basis, $T$ may be put in a diagonal block form, with blocks $D_{1}, \cdots, D_{r}$ on the diagonal, the dimension of each 
block $D_{j}$ being equal to the multiplicity $p_{j}$ of the corresponding real eigenvalue $t_{j} ; D_{j}$ is in superdiagonal form with $t_{j}$ 's on the diagonal. More specifically, $D_{j}$ may decompose into a diagonal block $t_{j} I$ of dimension $s_{j}<\left(p_{j}-1\right)$, and a block $D_{j}^{\prime}$ which has only one eigenvector and cannot be made diagonal. The corresponding family $F$ to such a $T$ is composed of associated pencils and skew-pencils, one for each block $t_{j} I$, $D_{j}^{\prime}$. In case at least one $D_{j}$ cannot be made diagonal, $F$ will be called a skew quasi-pencil.

5.1 THeOREM. A quasi-pencil or skew quasi-pencil $F$ is primary, and simply covers all of $E_{n+1}$ except the set of horizontal hyperplanes $\left\{z_{n+1}=\left(a t_{j}-b\right) /\left(t_{j}-1\right)\right\}$, where $t_{j}, j=1, \cdots, r$, are the distinct real eigenvalues of $T$.

Proof. If $F$ is to contain a line in the direction of a non-horizontal unit vector $\left(\lambda_{1}, \cdots, \lambda_{n+1}\right)$, then there must exist $x, y$ such that $(y-x)=$ $(T-I) x=k\left(\lambda_{1}, \cdots, \lambda_{n}\right)$, where $k \lambda_{n+1}=(b-a)$. Since $F$ is not a stack, we have that 1 is not an eigenvalue, $|T-I| \neq 0$, and there exists a unique solution for $x$. Since $y=T x+u$ is single valued for each point $(x ; a)$ in the hyperplane $H, H$ is simply covered. Any other point $\left(z ; z_{n+1}\right)$ in $E_{n+}$ will be covered if there exists an $x$ such that

$$
(z-x)=k(y-x),\left(z_{n+1}-a\right)=k(b-a) .
$$

For this we must have

$$
k(T-I)(x-w)+(x-w)=[k T-(k-1) I](x-w)=(z-w) .
$$

A unique solution for $(x-w)$ exists if $(k-1) / k$ is not an eigenvalue of $T$. We have

$$
k-1=\frac{\left(z_{n+1}-a\right) /(b-a)-1}{\left(z_{n+1}-a\right) /(b-a)}=\frac{z_{n+1}-b}{z_{n+1}-a} .
$$

Comparing with (4.1), we see that a unique solution for $x$ exists for all points $\left(z ; z_{n+1}\right)$ not in the horizontal hyperplanes containing the centers of the associated pencils and skew pencils.

6. Complex eigenvalues. In any odd dimensional space $E_{n+1}$, for an affine family $F$ such that the eigenvalues of $T$ are all complex, we have the following theorem.

6.1 THEOREM. Any affine family $F$, in $(n+1)$ dimensional space $E_{n+1}, n$ even, such that the transformation $T$ has no real eigenvalue, is primary and simple. That is, $F$ contains no horizontal line, contains exactly one line in every non-horizontal direction, no pair of lines of $F$ 
intersect, and each point of $E_{n+1}$ is covered by exactly one line of $F$.

Proof. In the proof of Theorem 5.1, under the present hypotheses, the determinant $|k T-(k-1) I|$ vanishes for no $k \neq 0$, so there is a unique line which covers each point $\left(z ; z_{n+1}\right)$ not in $H$. Each point $(x ; a)$ in $H$ also is uniquely covered since $y=T(x-w)+w$ is single valued. As in the proof of Theorem 5.1, since the determinant $|T-I|$ is not zero, we conclude that there is exactly one line of $F$ in every non-horizontal direction.

In any even dimensional space $E_{n+1}$, for an affine family $F$, not a stack, such that $T$ has only one real eigenvalue, we have the following theorem.

6.2 Theorem. Any affine family $F$, not a stack, in $(n+1)$ dimensional space $E_{n+1}, n$ odd, such that the transformation $T$ has only one real eigenvalue $t_{1}$, is primary, and simply covers all of $E_{n+1}$ except the hyperplane

$$
z_{n+1}=w_{n+1}=\frac{a t_{1}-b}{t_{1}-1}
$$

An $(n-1)$ dimensional flat $R$ in the hyperplane $z_{n+1}=w_{n+1}$ is infinitely covered, and the rest of the hyperplane is not covered, by $F$. The family $F$ is composed of an associated planar pencil, and of an associated simple family as in Theorem 6.1, of dimension $n$.

Proof. Since $F$ is not a stack, $|T-I| \neq 0$, and as in the proof of Theorem 5.1, we conclude that $F$ is primary. For any point $\left(z ; z_{n+1}\right)$ with $z_{n+1} \neq w_{n+1}$, so that $k \neq 1 /\left(1-t_{1}\right)$, the determinant $|k T-(k-1) I|$ does not vanish, so there is a unique line of $F$ which covers $\left(z ; z_{n+1}\right)$. Let an eigenvector $\tau_{1}$ corresponding to $t_{1}$ be chosen as the first vector of a basis. Then as shown in [5], the remaining basis vectors may be chosen so that $T$ assumes the form $\left(\begin{array}{cc}t_{1} & 0 \\ 0 & V\end{array}\right)$, where $V$ has only complex eigenvalues. For $k=1 /\left(1-t_{1}\right)$, the matrix $[k T-(k-1) I]$ has all zeros in it first column and first row. Accordingly $[k T-(k-1) I](x-w)=(z-w)$ has a solution only for vectors $(z-w)$ with $z_{1}=w_{1}$; for such vectors the solution for $\left(x_{2}-w_{2}\right), \cdots,\left(x_{n}-w_{n}\right)$ is unique, but $\left(x_{1}-w_{1}\right)$ is arbitrary. Thus for each point $x$ on the line $-\infty<x_{1}<\infty, x_{2}=w_{2}, \cdots, x_{n}=w_{n}$ in $H$, there is a line of $F$ through $x$ which covers the point $\left(w_{1}, z_{2}, \cdots, z_{n} ; w_{n+1}\right)$ of the hyperplane $z_{n+1}=w_{n+1}$. Therefore the $(n-1)$ dimensional flat $R$ defined by $z_{1}=w_{1}$ in the hyperplane is infinitely covered, and the rest of the hyperplane is not covered at all, by $F$.

It has been seen that the equation for any affine family, not a stack, 
can be put in the form $(y-w)=T(x-w)$. The origin in $E_{n+1}$ may be translated by a vector $(w ; 0)$. With respect to the new origin, the family has equation $y=T x$. Thus the most general affine family, $y=T x+u$, which is not a stack, may be obtained simply by translation of the family having equation $y=T x$. Accordingly in the remainder of this section and in the next, we take the equation for $F$ in the homogeneous form $y=T x$.

In case $T$ has several real eigenvalues different than 1 , and at least one pair of conjugate complex eigenvalues, then the basis may be chosen so that $T$ has block diagonal form, with a block $D_{i}$ on the diagonal for each real eigenvalue $t_{i} \neq 0$, and a block $Q_{j}$ for each pair of conjugate complex eigenvalues $\left(x_{j} \pm i y_{j}\right)$. (See [5].) The real blocks $D_{i}$ have already been described in $\S 5$. Each complex block $Q_{j}$ is of dimension $2 s_{j}$, where $s_{j}$ is the multiplicity of $\left(x_{j} \pm i y_{j}\right)$, and has $s_{j}$ two dimensional blocks $\left(\begin{array}{rr}x_{j} & y_{j} \\ -y_{j} & x_{j}\end{array}\right)$ on its diagonal, elements of $Q$, below the diagonal being zeros. The family $F$ corresponding to $T$ therefore is composed of associated pencils, skew pencils, and simple families as in Theorem 6.1.

In summary, the family $F$ corresponding to a matrix $T$ is a pencil if and only if the eigenvalues of $T$ are all real and equal; if $T$ has no real eigenvalue, $F$ is primary and simple; in any other case $F$ is primary, and simply covers all of $E_{n+1}$ except points in the set of hyperplanes

$$
\left\{z_{n+1}=w_{n+1, j}=\left(a t_{j}-b\right) /\left(t_{j}-1\right)\right\}, j=1, \cdots, p,
$$

where $t_{1}, \cdots, t_{p}$ are the distinct real eigenvalues of $T$. If the associated family of dimension $\left(p_{j}+1\right)$, where $p_{j}$ is the multiplicity of $t_{j}$, infinitely covers a flat of dimension $\left(p_{j}-1-q_{j}\right)$, then in the hyperplane $z_{n+1}=w_{n+1, j}$, a flat of dimension $\left(n-1-q_{j}\right)$ is infinitely covered by $F$; the remainder of each hyperplane is not covered by $F$.

7. Composition of general associated families. Any family $F$ in $E_{n+1}$ which is the composite of associated general families (families not necessarily corresponding to a linear transformation $T$ ), $F_{p}$ and $F_{n-p}$, in $E_{p+1}$ and $E_{n-p+1}$, is primary if $F_{p}$ is primary in $E_{p+1}$ and $F_{n-p}$ is primary in $E_{n-p+1}$. For by hypothesis there exists a unique $\left(x_{1}, \cdots, x_{p}\right)$ such that $\left(y_{i}-x_{i}\right)=k \lambda_{i}, i=1, \cdots, p$, and a unique $\left(x_{p+1}, \cdots, x_{n}\right)$ such that $\left(y_{j}-x_{j}\right)=k \lambda_{j}, j=p+1, \cdots, n$, where $k \lambda_{n+1}=(b-a)$, for any non-horizontal direction $\left(\lambda_{1}, \cdots, \lambda_{n+1}\right)$. If further both $F_{p}$ and $F_{n-p}$ are covering and simple (like the family of Theorem 6.1 ), then the composite family $F$ is covering and simple. For by hypothesis there exists a unique

$$
\left(x_{1}, \cdots, x_{p}\right)
$$

such that 


$$
\left(z_{i}-x_{i}\right)=k\left(y_{i}-x_{i}\right), \quad i=1, \cdots, p,
$$

and a unique

$$
\left(x_{p+1}, \cdots, x_{n}\right)
$$

such that

$$
\left(z_{j}-x_{j}\right)=k\left(y_{j}-x_{j}\right), \quad j=p+1, \cdots, n,
$$

where

$$
k(b-a)=\left(z_{n+1}-a\right),
$$

for any point $\left(z ; z_{n+1}\right)$ of $E_{n+1}$.

If however some point $\left(z_{1}, \cdots, z_{p} ; z_{n+1}\right)$ of $E_{p+1}$ is multiply covered by $F_{p}$, and if $F_{n-p}$ is covering, then since $F_{n-p}$ covers all points $\left(z_{p+1}\right.$, $\left.\cdots, z_{n} ; z_{n+1}\right)$ where $z_{p+1}, \cdots, z_{n}$ are arbitary, the composite family $F$ multiply covers all points $\left(z_{1}, \cdots z_{p}, z_{p+1}, \cdots, z_{n} ; z_{n+1}\right)$ of an $(n-p)$ dimensional flat. If $F_{p}$ does not cover some point $\left(z_{1}, \cdots, z_{p} ; z_{n+1}\right)$, then similarly there is an $(n-p)$ dimensional flat in $E_{n+1}$ which is not covered by $F$. Therefore no family $F$ other than a pencil, which is composed of associated families which are not simple, can be outwardly simple; any outwardly simple family which is composite must be either a pencil or simple. (For completion of the justification of this statement, see the following paragraph.)

Given two representative, outwardly simple families $F_{p}, F_{n-p}$, we may compose the primary sub-families (of all non-horizontal lines of $F_{p}$, $\left.F_{n-p}\right)$, to obtain a family $F$ which does not cover $(n-p)$ flats consisting of all points of the form $\left(z_{1}, \cdots z_{p}, z_{p+1}, \cdots z_{n} ; z_{n+1}\right),\left(z_{p+1}, \cdots, z_{n}\right)$ arbitrary, where $\left(z_{1}, \cdots, z_{p} ; z_{n+1}\right)$ is a point of $E_{p+1}$ which is covered only by an omitted horizontal line of $F_{p}$, and $p$ flats consisting of all points of the form $\left(z_{1}, \cdots z_{p}, z_{p+1}, \cdots z_{n} ; z_{n+1}\right),\left(z_{1}, \cdots, z_{p}\right)$ arbitrary, where $\left(z_{p+1}, \cdots\right.$, $\left.z_{n} ; z_{n+1}\right)$ is a point of $E_{n-p+1}$ which is covered only by an omitted horizontal line of $F_{n-p}$. In case there is a one-to-one correspondence of uncovered $(n-p)$ flats and uncovered $p$ flats, such that each corresponding pair of flats have the same values of $z_{n+1}$, then each such pair of corresponding flats together span a hyperplane in $E_{n+1}$. If $n$-dimensional covering line families are added in each of the hyperplanes, then the extended family $F$ covers all of $E_{n+1}$. If the number of such hyperplanes is finite or denumerable, it may be possible to choose such covering horizontal families in the hyperplanes that the covering extended family $F$ is representative. (See [6].) The extended family $F$ can be outwardly simple, however, only in case there is just one hyperplane and the associated families $F_{p}, F_{n-p}$ are pencils with common $w_{n+1}$, in which case $F$ neces- 
sarily is a pencil.

8. Generalization to Banach spaces. Some of the results of the preceding sections may be carried over to Banach spaces. If $f(x)$ is any non-vanishing bounded linear functional on a Banach space $B$, then

$$
H=[x \in B \mid f(x)=a] \text { and } K=[y \in B \mid f(y)=b]
$$

are hyperplanes which are parallel to the closed linear subspace $E=$ $[x \in B \mid f(x)=0]$. The space $B$ may be the Cartesian product of any Banach space $E$ and the real number line; for such a product a bounded linear functional $f$ always exists having $E$ for its null subspace.

There is an $\alpha$ in $B$ such that $f(\alpha)=\|\alpha\|=1$. If $P$ is any point of $B$, we have $P=f(P) \cdot \alpha+[P-f(P) \cdot \alpha] ;[P-f(P) \cdot \alpha]$ is in the null subspace $E$ of $f$. If also $P=z_{f} \cdot \alpha+z$, with $z$ in $E$, we have $f(P)=z_{f}, 0=P$ $-f(P) \cdot \alpha-z$, or $z=P-f(P) \cdot \alpha$. Thus with respect to any fixed "vertical " vector $\alpha$, any point $P$ in $B$ has unique coordinates $\left(z ; z_{f}\right)$. A direction $\left(v ; v_{f}\right)$ is " horizontal" if $f\left(v ; v_{f}\right)=v_{f}=0$.

As in the finite dimensional case, the equation for any pencil of lines in $B$ is $(y-w)=c I(x-w)$, where $I$ is the identity transformation in $E$ and $c \neq 1$. To show this, let the origin of $B$ be translated from $(0 ; 0)$ to $(w ; 0)$. Then the translated family of lines has equation $y=c I x$. Define

$$
w_{f}=a-\begin{gathered}
b-a \\
c-1
\end{gathered} .
$$

Points $z$ on the line through $(x ; a)$ and $(y ; b)$, where $y=c I x$, are given by $e(x ; a)+(1-e)(y ; b)$. There is a unique $e$ such that $e a+(1-e) b=w_{f}$, namely

$$
e=\frac{w_{f}-b}{a-b},
$$

and

$$
e x+(1-e) y=[e+(1-e) c] x=0 x=0 .
$$

Therefore all lines of the family pass through the point $\left(0 ; w_{f}\right)$. Conversely for any non-horizontal direction $\left(v ; v_{f}\right)$, there exist a unique $x$ and $y=c x$ in $E$ such that

$$
(y-x)=(c-1) x=k v,(b-a)=k v_{f} ;
$$

thus the family contains one line through $\left(0 ; w_{f}\right)$ in every non-horizontal direction, and is made into a pencil, with center $\left(0 ; w_{f}\right)$, by addition of all horizontal lines through $\left(0 ; w_{f}\right)$. 
If the affine family $y=T x+u$, where $T$ is a not necessarly bounded linear transformation, is not a stack, then 1 must belong either to the resolvent set, or to the continuous or residual spectrum of $T$. (See [3, p. 31].) In case $u$ is in the domain of $(T-I)^{-1}$, the corresponding family may be translated so that the equation becomes $y=T x$. Replacement of reference hyperplane

$$
K=\left[\left(z ; z_{f}\right) \in B \mid z_{f}=b\right]
$$

by

$$
K^{\prime}=\left[\left(z ; z_{f}\right) \in B \mid z_{f}=b+h(b-a)\right]
$$

does not change the family of lines, but induces replacement of $T$ by $T^{\prime}=(1+h) T-h I$. Thus an eigenvalue $\lambda^{\prime}$ of $T^{\prime}$ corresponds to an eigenvalue

$$
\lambda=\frac{h+\lambda^{\prime}}{h+1}
$$

of $T$; in particular if 0 is an eigenvalue of $T$, it may be replaced by any desired value $\lambda^{\prime}$ except 1 by taking $h=-\lambda^{\prime}$. The choice $h=-1$ is impossible since $K^{\prime}$ then would coincide with $H$; an eigenvalue 1 of $T$ is preserved under this transformation.

The affine family $y=T x$, where $T$ is a not necessarily bounded linear transformation, if not a stack, will contain exactly one line in every non-horizontal direction $\left(v ; v_{f}\right)$ where $v$ is in the domain of $(T-I)^{-1}$. This follows since the system $(y-z)=(T-I) x=l v v$, where $k v_{f}=(b-a)$, then has a unique solution for $x$. If $U$ is a bounded, one-to-one linear transformation on all of $E$ to all of $E$, then by a theorem of Banach, $U$ is an isomorphism, so the affine family $F$ corresponding to $T=U+I$ is primary; more generally $F$ is primary for any bounded or unbounded $U$ which is linear and one-to-one on $E$ to all of $E$. If the domain $D$ of $U$ is not all of $E$, then $T=U+I$ also will be defined only on $D$, so that $F$ is primary but covers only the proper subset $(D ; a)$ of hyperplane $H$.

The affine family will simply cover a point $\left(z ; z_{f}\right)$ in $B$ if the system $(z-x)=k(y-x)$, or $z=[k T+(1-k) I] x$, where $k(b-a)=\left(z_{f}-a\right)$, has a unique solution for $x$. This will be the case for all $\left(z ; z_{f}\right)$ in every hyperplane $z=z_{f}$ such that $(1-k) / k=-\left(b-z_{f}\right) /\left(a-z_{f}\right)$ is not in the point spectrum of $T$, and such that $z$ is in the domain of $[k T+(1-k) I]^{-1}$.

\section{REFERENCES}

1. P. C. Hammer, Diameters of convex bodies, Proc. Amer. Math. Soc., 5 (1954), 304306.

2. P. C. Hammer and A. Sobczyk, Planar line families I, II, Proc. Amer. Math. Soc., 4 (1953), 226-233, and 341-349. 
3. E. Hille, Functional analysis and semi-groups, Amer. Math. Soc. Colloquim Publications 31 (1948).

4. Amasa Forrester, A theorem on involutory transformations, Proc. Amer. Math. Soc., 3 (1952), 333-334.

5. A. Sobczyk, Canonical form for a real matrix, unpublished manuscript.

6. A. Sobczyk, Families of lines, to be submitted to Mem. Amer. Math. Soc.

UNIVERSITY OF FLORIDA 


\section{PACIFIC JOURNAL OF MATHEMATICS}

\section{EDITORS}

\author{
H. L. Royden \\ Stanford University \\ Stanford, California \\ E. Hewitt \\ University of Washington \\ Seattle 5 , Washington
}

R. P. Dilworth

California Institute of Technology

Pasadena 4, California

\author{
A. HorN* \\ University of California \\ Los Angeles 24, California
}

\section{ASSOCIATE EDITORS}

\author{
E. F. BECKENBACH \\ C. E. BURGESS \\ H. BUSEMANN \\ H. FEDERER
}

\author{
M. HALL \\ P. R. HALMOS \\ V. GANAPATHY IYER \\ R. D. JAMES
}

\author{
M. S. KNEBELMAN \\ I. NIVEN \\ T. G. OSTROM \\ M. M. SCHIFFER
}

J. J. STOKER

G. SZEKERES

F. WOLF

K. YOSIDA

\section{SPONSORS}

\author{
UNIVERSITY OF BRITISH COLUMBIA \\ CALIFORNIA INSTITUTE OF TECHNOLOGY \\ UNIVERSITY OF CALIFORNIA, BERKELEY \\ UNIVERSITY OF CALIFORNIA, DAVIS \\ UNIVERSITY OF CALIFORNIA, LOS ANGELES \\ UNIVERSITY OF CALIFORNIA, SANTA BARBARA \\ MONTANA STATE UNIVERSITY \\ UNIVERSITY OF NEVADA \\ OREGON STATE COLLEGE \\ UNIVERSITY OF OREGON \\ UNIVERSITY OF SOUTHERN CALIFORNIA
}

STANFORD RESEARCH INSTITUTE

STANFORD UNIVERSITY

UNIVERSITY OF UTAH

WASHINGTON STATE COLLEGE

UNIVERSITY OF WASHINGTON

AMERICAN MATHEMATICAL SOCIETY HUGHES AIRCRAFT COMPANY SHELL DEVELOPMENT COMPANY

Mathematical papers intended for publication in the Pacific Journal of Mathematics should be typewritten (double spaced), and the author should keep a complete copy. Manuscripts may be sent to any of the editors. Manuscripts intended for the outgoing editors should be sent to their successors. All other communications to the editors should be addressed to the managing editor, Alfred Horn at the University of California, Los Angeles 24, California.

50 reprints of each article are furnished free of charge; additional copies may be obtained at cost in multiples of 50 .

The Pacific Journal of Mathematics is published quarterly, in March, June, September, and December. The price per volume (4 numbers) is $\$ 12.00$; single issues, $\$ 3.50$. Back numbers are available. Special price to individual faculty members of supporting institutions and to individual members of the American Mathematical Society: $\$ 4.00$ per volume; single issues, $\$ 1.25$.

Subscriptions, orders for back numbers, and changes of address should be sent to Pacific Journal of Mathematics, c/o University of California Press, Berkeley 4, California.

Printed at Kokusai Bunken Insatsusha (International Academic Printing Co., Ltd.), No. 10, 1-chome, Fujimi-cho, Chiyoda-ku, Tokyo, Japan.

* During the absence of E. G. Straus.

PUBLISHED BY PACIFIC JOURNAL OF MATHEMATICS, A NON-PROFIT CORPORATION COPYRIGHT 1956 BY PACIFIC JOURNAL OF MATHEMATICS 


\section{Pacific Journal of Mathematics}

\section{Vol. 6, No. $3 \quad$ BadMonth, 1956}

Richard Arens and James Eells, Jr., On embedding uniform and topological spaces ............................................ 397

N. Aronszajn and Prom Panitchpakdi, Extension of uniformly continuous transformations and hyperconvex metric spaces .............. 405

Kai Lai Chung and Cyrus Derman, Non-recurrent random walks ........ 441

Harry Herbert Corson, III, On some special systems of equations . . . . . . . . . 449

Charles W. Curtis, On Lie algebras of algebraic linear transformations . . . 453

Isidore Heller, Neighbor relations on the convex of cyclic permutations . . . . 467

Solomon Leader, Convergence topologies for measures and the existence of transition probabilities..................................... 479

D. H. Lehmer, On certain character matrices ...................... 491

Michael Bahir Maschler, Minimal domains and their Bergman kernel function ........................................... 501

Wm. M. Myers, Functionals associated with a continuous transformation ................................... 517

Irving Reiner and Jonathan Dean Swift, Congruence subgroups of matrix groups ....................................... 529

Andrew Sobczyk, Simple families of lines ................... 541

Charles Standish, A class of measure preserving transformations ........ 553 Jeremiah Milton Stark, On distortion in pseudo-conformal mapping ..... 565 